\title{
Trends in Internal Medicine
}

\section{Hidden Horror Challenges for Oral Mucormycosis with COVID 19 Patients Case Series Original Study}

\section{Dr. Rawaa Y. Al-Rawee', Dr. Ali Mohammad Saeed², Dr. Ahmed khaldoon Tariq Alomari², Dr. Bashar Abdul-Ghani Tawfeeq ${ }^{1}$, and Dr. Saud Salim Saeed ${ }^{1}$}

${ }^{1}$ Al-Salam Teaching Hospital. Mosul, Iraq.

${ }^{2}$ Al-Jumhory Teaching Hospital. Mosul, Iraq.

\section{"Correspondence:}

Rawaa Y. Al-Rawee, BDS. M.Sc OS., MOMS FDS RCPS Glasgow, Ph.D. MaxFacs. Consultant, Department of Oral and Maxillofacial Surgery, Al-Salam Teaching Hospital. Mosul, Iraq.

Received: 30 August 2021; Accepted: 02 October 2021

Citation: Rawaa Y. Al-Rawee, Saeed AM, Alomari AKT, et al. Hidden Horror Challenges for Oral Mucormycosis with COVID 19 Patients Case Series Original Study. Trends Int Med. 2021; 1(2): 1-6.

\begin{abstract}
Purpose: Explore the early identification significance of mucormycosis in Covid 19 patients and highlighting the role of MDT cooperation in reducing mortality and morbidity rate.

Material and Method: Forty-three patients are included in this study as mucormycosis infection with diabetes mellitus (MID) divided in 2 groups: group 1: 15 patients recorded to have mucormycosis infection with diabetes mellitus only (MID), group 2: 28 patients recorded to have previous Covid attack added to mucormycosis infection with diabetes mellitus (CMID).

Result: Mann-Whitney Test and Wilcoxon W test are used for detection of significant differences between 2 groups where $z$ value considers less than 0.5 to be significant. Severity and stages confirm high significant difference between MID and CMID (0.042, 0.000) while prognosis confirm no significant differences $(0.521)$.

Conclusion: Diabetic patients are more prone to have viral and bacterial infections make them need special care. COVID-19 with the immune dysregulation is concomitant with a significant prevalence of fungal infections as secondary attacks; early diagnosis and management can help in reduce subsequently mortality and morbidity.
\end{abstract}

\section{Keywords}

Mucormycosis, Naso-cerebral mucormycosis, Diabetes mellitus, Covid 19 attacks complications, Ketoacidosis.

\section{Introduction}

Mucormycosis, widely known to be most serious life-threatening fungal infection virtually affecting diseased individuals in most countries in spite of industrial developments [1].

Phagocytosis is the most host defense mechanism clinically evident and proved against mucormycosis; based on those immunecompromised patients are most commonly affected individual as:

- About $70 \%$ of attacks affecting poorly controlled diabetes mellitus, hyperglycemia, or diabetic ketoacidosis [2].

- Hematological malignancies or persistent neutropenia.

- Hematopoietic stem cell transplant recipients,

- Host diseases receiving high-dose corticosteroids for graft.

- Solid organ transplant recipients receiving continuous intensive immunosuppression

- Patients with severe burns or trauma are at risk for cutaneous mucormycosis

- Gastrointestinal mucormycosis has been reported in patients who have ingested contaminated food, fermented beverages, or herbal products [3].

Vessel thrombosis end with tissue necrosis are the most clinical 
sequence can be seen in such patients; serious dissemination affecting valuable organs with short and fast time make the mucormycosis infection attack as life threatening condition with challenge in urgent diagnosis and treatment. Brand etal 2005 stated that "In most cases, the infection is relentlessly progressive and results in death unless treatment with a combination of surgical debridement and antifungal therapy is initiated promptly." [1].

"Rhino-cerebral mucormycosis continues to be the most common form of the disease, accounting for between one-third and one-half of all cases of mucormycosis" [4] Sinusitis or peri-orbital cellulitis are the most reliable initial symptoms occur $[5,6]$ beside facial pain, numbness, followed by the onset of eye complication as conjunctival suffusion, blurry vision, and soft tissue swelling [79]. Half of cases have no fever [6] eventually chemosis, proptosis, ophthalmoplegia and vision loss in untreated cases can present; bilateral eye involvement can show sign of cavernous sinus thrombosis and cranial extension.

Challenge in diagnosis is manifested upon visual inspection, specifically the normal appearance of the fungal spread in infected tissues. Final formation of the black necrotic escher results from blood vessel thrombosis and tissues infarction $[10,11]$.

There are no reliable serologic or skin tests for mucormycosis. Therefore, the diagnosis should be made by biopsy of infected tissues [12].

In this extraordinary epidemic attack of Coronavirus disease 2019 (COVID-19); many serious end stage patients are diagnosed with fast progressive deterioration of mucormycosis infection attack in concomitant with Covid attack or post Covid attack [13].

Briefly Covid 19 is a new disease entity caused by a novel coronavirus (SARS-CoV-2) first documented in China in December 2019 and subsequently causing a worldwide pandemic.

Viral pathophysiology is still not clear and under research, beside that many medical literatures emphasize various sign and symptom manifested as serious complications of the disease as acute respiratory failure, acute respiratory distress syndrome (ARDS), acute liver injury, acute cardiac injury, acute kidney injury, secondary infection and septic shock [14].

Mucormycosis and orbital compartment syndrome are rare, time sensitive conditions that must be recognized and treated promptly to avoid mortality and morbidity [15].

There is no evident co-relation understands between Covid attack and increase risk and progressivity of mucormycosis are highlighted in articles.

The study was first of its kind, and minimum literature was available for comparison. Authors suspect the presence of difference in approaching patients. The survival rate can be improved in cases in which early detection and complete treatment are possible.

\section{Objectives}

In this comparative clinical study authors aims to explore the early identification significance of mucormycosis in Covid 19 patients. Discuss difference in management protocol between Covid and non Covid patients. Generally authors aim to highlighting the role of MDT cooperation in reducing mortality and morbidity rate, and maxillofacial surgeon role in early diagnosis and surgical treatment too.

\section{Material and Methods}

Maxillofacial units in both Al-Salam and Al-Jumhore Teaching Hospitals receive about more than 100 immunocompromised patients monthly complain from serious odontogenic infection which needs massive urgent medical and surgical interventions.

All work is approved by the scientific committee of Nineveh Health Directorate / MOH / Iraq by licenses' Number (3646) in date (31/1/2021) and all participants signed an informed consent agreement. This study followed the Declaration of Helsinki on medical protocol and ethics, and the regional Ethical Review committee approved the study.

\section{Inclusion and Exclusion criteria \\ Inclusion Criteria}

- Patients diagnosed as Mucormycosis and COVID 19.

- Patients are immune-compromised (Diabetic)

\section{Exclusion Criteria}

- Patients diagnosed as other infections or suspected mucormycosis.

- Patients are immune-compromised (rather than Diabetic)

- Patients with poor follow up

Forty-three patients are included in this study as mucormycosis infection with diabetes mellitus (MID) divided in 2 groups.

Group 1: 15 patients recorded to have mucormycosis infection with diabetes mellitus only (MID).

Group 2: 28 patients recorded to have previous Covid attack added to mucormycosis infection with diabetes mellitus (CMID)

Patients are shared with the same presentation and complain but differ in severity, time of ask treatment, clinical extension, date of diagnosis and treatment start. Sever swelling in the maxillary posterior region are the general manifestation of all patients.

Group 1: Mucormycosis Infection with Diabetes Mellitus (MID) Description.

15 Patients are collected in the 5 years period, given same history and complain of multiple recurrent ignored attacks of odontogenic pain, fever and antibiotics cover show no response eventually end with rapid progressive deteriorations. Clinically the presence of hyper glycaemia reflects the characters of uncontrolled diabetes. General clinical examinations reflect manifestation as fever, hypotensive, conscious and tired. 
Extra oral examination show sever swelling affecting maxillary bone and sinus with or with no orbital extension, Headache, pain, with ophthalmologic symptoms ranging from pain and proptosis to ophthalmoplegia and blindness. Shiny red skin in some places is manifested.

Intraoral examination shows various sign and symptom from simple teeth mobility with redness of the gingiva, badly brokendown teeth, pus discharge in some cases, to more sever necrosis and palatal black patches with different level of extensions (A black necrotic eschar).

\section{Haematological Investigation and examination:}

Blood sample include complete blood counts, blood urea, serum creatinine, serum glucose. Radiographical investigation and examination (OPG, MRI, CT scan) show dissimilar level of bone destruction according to severity, opacification of sinuses may be observed in conjunction with patchy effacement of bony walls of sinuses.

Basically, diagnosis confirmed by incisional biopsy to excluding other types of infections or disease. Clinical differential diagnosis of lesion should include squamous cell carcinoma, chronic granulomatous infection like tuberculosis, midline lethal granuloma and other deep fungal infections.

Patients hospitalized for treatment and controlling underlying disease in the internal medicine department. Rigid nasal endoscopy not performed for patients as extension and stage are clear with other investigations. Neurosurgical and ophthalmological consultation also performed for patients on need according to severity and extensions.

When diagnosis confirmed presences of mucormycosis infection so start firstly with conservative treatment protocol which include amphotericin B $(0.8 \mathrm{mg} / \mathrm{kg} /$ day for 3 weeks $)$ use; control the blood sugar level as urgent as possible with bolus doses according to European Confederation of Medical Mycology [20].

Secondly prepare the patients for surgical intervention which include extensive debridement of the necrosed sequestrate bone (maxilla, nose, orbit and plate) under local or general anesthesia depend on multiple factors in different levels of extensions and approaches according to each case alone.

Patient observed closely for healing and discharged home after 2 weeks with follow up. Patients were followed closely for disease progression and repeated debridement was done when necessary. After 3-4 months of follow-up period; prosthetic reconstruction are managed. Unfortunately two cases died and 13 cases going on their lives.

\section{Group 2: COVID 19 / Mucormycosis Infection with Diabetes Mellitus (MID) Description}

Group 2: 28 patients collected in six months only, recorded to have previous Covid attack added to mucormycosis infection with diabetes mellitus (CMID).

Same protocol was followed in recording these cases (clinical, radiographical, diagnosis and management).

Records of each patient include: patientidentity code, demographical data, and symptom onset to presentation to maxillofacial unit. Stage identifications divided to stage I (sino-nasal stage), stage II (rhino-orbital stage), stage III (rhino-obito-cerebral stage) according to previous study done by Ahmed Al-Abbasi 2017 in Iraq Basra $[16,17]$. Severity also divided to three grades stage one mild, stage 2 considered moderate, stage three were extension are sever grade. Also, days need to diagnose, consultation, time to start of antifungal therapy (days) from diagnosis, surgical intervention from diagnosis and lastly prognosis.

Rigid nasal endoscopy also performed primarily to detect extension, staging and as well as therapeutic in some cases.

\section{Ethical Approval}

All work is approved by the scientific committee of Nineveh Health Directory / MOH / Iraq which follows the Helsinki Declaration.

\section{Result}

By use of SPSS version 25 statistical analyses was done. Table 1 highlights the descriptions analysis differences between the two groups (MID + CMID) includes age groups, gender, stages, severity and fate of patients involved in the study. Differences are shown in age grouping; patients aged between (51-60 years, 40\%) in MID group while (41-50 years, 35\%) in CMID group.

Table 1: Descriptive Analysis of Both Groups.

\begin{tabular}{|c|c|c|c|c|c|}
\hline \multirow{2}{*}{\multicolumn{2}{|c|}{ Frequency }} & \multicolumn{2}{|c|}{ * Group 1} & \multicolumn{2}{|c|}{ ** Group 2} \\
\hline & & \multirow{2}{*}{$\begin{array}{c}\text { No. of } \\
\text { Patients } \\
1\end{array}$} & \multirow{2}{*}{$\begin{array}{c}\text { Percentage } \\
6.66\end{array}$} & \multirow{2}{*}{$\begin{array}{c}\text { No. of } \\
\text { Patients } \\
2\end{array}$} & \multirow{2}{*}{$\begin{array}{c}\text { Percentage } \\
7.14\end{array}$} \\
\hline \multirow{4}{*}{ Age group } & $30-40$ & & & & \\
\hline & $41-50$ & 4 & 26.66 & 10 & 35.7 \\
\hline & $51-60$ & 6 & 40 & 8 & 28.5 \\
\hline & Above 60 & 4 & 26.66 & 8 & 28.5 \\
\hline \multirow{2}{*}{ Gender } & Male & 8 & 53.3 & 11 & 39.3 \\
\hline & Female & 7 & 46.7 & 17 & 60.7 \\
\hline \multirow{3}{*}{ Stages } & I & 9 & 60.0 & 3 & 10.7 \\
\hline & II & 4 & 26.7 & 7 & 25.0 \\
\hline & III & 2 & 13.3 & 18 & 64.3 \\
\hline \multirow{3}{*}{ Severity } & Mild & 9 & 60 & 7 & 25.0 \\
\hline & Moderate & 3 & 20 & 10 & 35.7 \\
\hline & Sever & 3 & 20 & 11 & 39.3 \\
\hline \multirow{2}{*}{ Fate } & Live & 13 & 86.7 & 22 & 78.6 \\
\hline & Dead & 2 & 13.3 & 6 & 21.4 \\
\hline
\end{tabular}

* Group 1: MID (Total Patients Number $=15$ in 5 years period)

** Group 2: CMID (Total Patients Number $=28$ in 6 Months Period)

Gender show differences too; male (53.3\%) more affected in MID than Female while inversed in CMID cases (female 60\%).

High divergences are clear in both staging and severity stage one $(60 \%)$ with mild severity $(60 \%)$ are recorded in MID group in 
contrast CMID cases are with stage three (64.3\%) and moderate to severe state $(35.7,39.3)$.

Prognosis of patients are also varied with mortality rate $21.4 \%$ in CMID while $13.3 \%$ in MID. Mann-Whitney Test and Wilcoxon $\mathrm{W}$ test are used for detection of significant differences between 2 groups where $\mathrm{z}$ value considers less than 0.5 to be significant. Severity and stages confirm high significant difference between MID and CMID $(0.042,0.000)$ while prognosis confirm no significant differences (0.521) (Table 2).

Table 2: Mann-Whitney and Wilcoxon W Test for significant Differences.

\begin{tabular}{|l|c|c|c|}
\hline Test Statistics $^{\text {a }}$ & Stage & Severity & Fate \\
\hline Mann-Whitney U & 77.500 & 135.000 & 193.000 \\
\hline Wilcoxon W & 197.500 & 255.000 & 313.000 \\
\hline Z & $-3.638-$ & $-2.029-$ & $-.643-$ \\
\hline Asymp. Sig. (2-tailed) & .000 & .042 & .521 \\
\hline a. Grouping Variable: Cod & & & \\
\hline
\end{tabular}

More over time difference are documented between the two groups. Time challenge in mucormycosis is important factor. For that reason author document the days from symptom onset before seeking help as shown in table 3. Days allocated into 5 categories and number of patients recorded.

Table 3: Time Sequences from Symptom Onset before Contact Professionals.

\begin{tabular}{|l|c|c|c|c|}
\hline \multirow{2}{*}{ Days } & \multicolumn{2}{|c|}{ MID } & \multicolumn{2}{c|}{ CMID } \\
\cline { 2 - 5 } & Days No. & $\%$ & Days No. & $\%$ \\
\hline $0-10$ days & 12 & 80 & & \\
\hline $11-20$ days & 1 & 10 & & \\
\hline $21-30$ days & 2 & 10 & 7 & 25 \\
\hline $31-60$ days & & & 18 & 64.28 \\
\hline Above 61 days & & & 3 & 10.71 \\
\hline
\end{tabular}

Prognosis of patient's treatment is recorded furthermore compared with time schedule. Death occurs in both groups and evaluated according to time (Table 4).

Table 4: Death and Time Evaluation.

\begin{tabular}{|l|c|c|}
\hline Days & MID & CMID \\
\hline $0-10$ days & & \\
\hline $11-20$ days & & \\
\hline $21-30$ days & 2 & 2 \\
\hline $31-60$ days & & 4 \\
\hline Above 61 days & & \\
\hline
\end{tabular}

\section{Discussion}

Mucormycosis known term used to describe a very serious invasive fungal infection affecting immunocompromised patients specially ketoacidotic diabetic patients. This type of infection caused by various members of the class phycomycetes. This type of fungi is widely spread in nature and rarely affecting immune-competent human being, on the contrary [18] seen usually in immunecompromised patients as acute leukemia, lymphoma, and diabetic ketoacidosis added to that patients receiving immune-suppressant treatment after organ transplantation [19].
In a global study by European Confederation of Medical Mycology perform guidelines and focus on four important points which includes

- Difficult to diagnose such rare disease present with high morbidity and mortality.

- Diagnosis is often delayed, and disease tends to progress rapidly.

- Urgent surgical and medical intervention is lifesaving.

- Guidance on the complex multidisciplinary management has potential to improve prognosis." [20].

All surgeons and physicians agreed on such important unique clinical features distinguished with mucormycosis infection. Although of rarity but challenge in diagnosis, wide rapid urgent spread and high morbidity mortality rate are serious.

In these extraordinary circumstances were the epidemic attack of COVID 19 are spread widely like wild fire, affecting all human being; immunocompromised patients also distressing in this situation. Authors observe that advanced sever cases of mucormycosis are increased post COVID 19 attacks; for that reason authors like to evident the multifactorial difference between mucormycosis infection severity and stages with prognosis in the period pre and post COVID 19 occurrence.

These factors include age, gender, severity, stages and fate or prognosis of patients. Co-relation differences are evaluated to specify if there is significant difference or not.

Limited articles highlighting this subject either as case reports or case series but there is no comparative studies in relation to our knowledge and searching which make it original article.

In the pre-Covid epidemic periods, various articles assessing; researcher approve that occurrence of mucormycosis has risen in developing countries [21].

Chakrabarti and Singh 2014 in his published article emphasize a three sequential mucormycosis case series have been reported from a single tertiary center in India: in different periods [(1992 1999 -10 years, 129 cases $)$, [(2000 - 2004 - 5 years, 178 cases $)]$ and [(2006 - 2007, an 18-month, 75 cases $)]$. Authors support the idea of increasing attacks with mucormycosis [22].

In this article only 15 cases are collected from maxillofacial departments in Mosul / Iraq for period of 5 years durations. These patients have mucormycosis infection synchronized in diabetes mellitus type II disease. Stage one (mild presentation) show $60 \%$ of total 15 patients with good prognosis $(86.7 \%)$ after medical and surgical management following the global published guidelines [20].

Periods of COVID attack show wide disturbances in most of countries (developed and developing societies together). Different unexpected complications are recorded in Covid patients. In this article twenty eight patients attain same maxillofacial departments in period of 6 months. Late sever disseminated manifestations (stage III 64.3\% + severe 39.3\%) are clear in these cases with bad 
prognosis as 6 patients $(21.4 \%)$ are died in spite of urgent medical and surgical management.

Complex interacted influences are present in COVID 19 cases that make them more prone to have secondary infection or fungal strike. It's crucial to be discussed, these are:

- Many international researches discuss the pathophysiologic features of COVID-19 specifically that which may facilitate secondary fungal infections such as extensive pulmonary disease susceptibility and the consequently alveolo-interstitial pathology with invasive fungal infections enhancement. [23].

- As well as dysregulation of the natural immune system which reflected through diminished number of T lymphocytes, CD4+T, and CD8+T cells [24]. Dexamethasone is recommended to be used in COVID 19 patients by the National Institute of Health especially in patients need ventilation or oxygen supplement [25]. "The guidelines specifically mention the risk of developing a secondary infection $[26,27] "$.

- Researches also emphasize the importance of hospital admission periods of COVID 19 patients which make them also prone to fungal infection more. This confirmed in recent published study by Rawson etal [28], stated that $8 \%$ of patients have hospitalacquired infections on the contrary $72 \%$ of patients use broadspectrum antibiotics with no evidence of underlying infection.

In two different recent studies stated that fungal infection with COVID can developed in bloodstream, patients show higher mortality $(53 \%, 60 \%)[29,30]$ Pakistan (15.6\%) and Italy (27.7\%) likewise developments of aggressive fungal infection show high incidences [31,32]. Limited cases are discussed in literature searching include term both COVID and mucormycosis cases this also add argument for the originality of this article.

Case report discussed by Hanley et al. 2020, the patient has COVID-19 with pneumonia along with infarction of middle cerebral artery prone to mucormycosis involving the lungs and brain was incidentally revealed through a postmortem study [33]. 2020 Mehta et al. reported case revealed diabetic ketoacidosis with COVID-19 infection with left-sided ptosis and proptosis [34].

European Confederation of Medical Mycology in their global guidelines emphasis that "mortality rates for mucormycosis range from $40 \%$ to $80 \%$ with varying rates depending on underlying conditions and sites of infection" in spite of the cause [35-39]. Also they were reporting that healthy immune state patients show high survival rates. Similarly, "earlier tissue-based diagnosis is often feasible and surgical debridement may result in cure".

According to what was previously reviewed, authors in this article confirm the seriousness of mucormycosis cases and the crucial early diagnosis (time sensitive) and urgent management. This proved through the significance difference between severity and stages. Late diagnosis can increase mortality rate with dissemination and spread of infection affecting dangerous area as orbital and brain extensions.
Diabetic patients who are infected with corona virus, it's preferable to follow them at least six months post attack and advised to seek help in any abnormal sign and symptom occurrence. Inform practitioners to take care and refer patients' high risk as soon as possible to avoid serious complications.

The cons of this study include increasing sample size, increase time of cases collection as well as cover more specialties as Ear Nose Throat branch cases, Ophthalmological branch cases and Neurosurgery cases. Finally, comparison can do in more centers in other cities.

\section{Conclusion}

Diabetic patients are more prone to have viral and bacterial infections make them need special care. COVID-19 with the immune dysregulation is concomitant with a significant prevalence of fungal infections as secondary attacks. Multifactorial causes can exacerbate this serious condition end with high mortality rate.

Mucormycosis is time sensitive condition; early diagnosis and management are important can help in reduce subsequently mortality and morbidity.

\section{References}

1. Spellberg B, Edwards J, Ibrahim A, et al. Novel Perspectives on Mucormycosis: Pathophysiology, Presentation, and Management. Clinical Microbiology Reviews. 2005; 18: 556-569.

2. McNulty JS: Rhinocerebral mucormycosis: predisposing factors. Laryngoscope. 1982; 92: 1140-1143.

3. Chinn RY, Diamond RD: Generation of chemotactic factors by Rhizopus oryzae in the presence and absence of serum: relationship to hyphal damage mediated by human neutrophils and effects of hyperglycemia and ketoacidosis. Infect. Immun.1982; 38: 1123-1129.

4. Pillsbury HC, Fischer ND: Rhinocerebral mucormycosis. Arch. Otolaryngol. 1977; 103: 600-604.

5. Dhiwakar M, Thakar A, Bahadur S. Improving outcomes in rhinocerebral mucormycosis-early diagnostic pointers and prognostic factors. J. Laryngol Otol. 2003; 117: 861-865.

6. Talmi YP, Goldschmied-Reouven A, Bakon M, et al. Rhinoorbital and rhino-orbito-cerebral mucormycosis. Otolaryngol. Head Neck Surg. 2002; 127: 22-31.

7. Khor BS, Lee MH, Leu HS, et al. Rhinocerebral mucormycosis in Taiwan. J. Microbiol. Immunol. Infect. 2003; 36: 266-269.

8. Peterson KL, Wang M, Canalis RF, et al. Rhinocerebral mucormycosis: evolution of the disease and treatment options. Laryngoscope. 1997; 107: 855-862.

9. Thajeb P, Thajeb T, Dai D. Fatal strokes in patients with rhinoorbito-cerebral mucormycosis and associated vasculopathy. Scand. J. Infect. Dis. 2004; 36: 643-648.

10. Husain S, Alexander BD, Munoz P, et al. Opportunistic mycelial fungal infections in organ transplant recipients: emerging importance of non-Aspergillus mycelial fungi. Clin. Infect. Dis. 2003; 37: 221-229. 
11. Petrikkos G, Skiada A, Sambatakou H, et al. Mucormycosis: ten-year experience at a tertiary-care center in Greece. Eur. J. Clin. Microbiol. Infect. Dis. 2003; 22: 753-756.

12. Al-Rawee R, Al-Bakry R, Khider H, et al. Worth of Time Challenge and Multi-Disciplinary Team in Rhino Maxillary Mucormycosis Mortality Detraction: Longitudinal Study in Al-Salam Teaching Hospital. Annals of Tropical Medicine \& Public Health S196. 2019; 22.

13. Werthman A. Mucormycosis with orbital compartment syndrome in a patient with COVID-19. American Journal of Emergency Medicine. Article in press. 2021; 42: 5-264.

14. WHO official site Coronavirus disease (COVID-19) advice for the public. last updated on 3 March 2021.

15. Prakash H, Chakrabarti A. Global Epidemiology of Mucormycosis. J. Fungi. 2019; 5: 26.

16. Al-Abbasi A, Abdil-Wahab A, Al-Jezani AR, et al. RhinoOrbito-Cerebral Mucormycosis in Basrah - IRAQ. Bas J Surg. 2017; 23: 9-14.

17. Singh VP, Bansal C, Kaintura M. Sinonasal Mucormycosis: A to Z. Indian J Otolaryngol Head Neck Surg. 2019; 71: 1962-1971.

18. Mignogna MD, Fortuna G, Leuci S, et al. Mucormycosis in immunocompetent patients: a case-series of patients with maxillary sinus involvement and a critical review of the literature. International Journal of Infectious Diseases. 2011; 15: 533-540.

19. Pagano L, Ricct P, Tonso A, et al. Mucomycosis in Patients with Haematological Malignancies a Retrospective Spective Clinical Study of 37 Cases.British Journal of Haematology 1997; 99: 331-336.

20. Cornely OA, Izquierdo AA, Arenz D, et al. Global guideline for the diagnosis and management of mucormycosis: an initiative of the European Confederation of Medical Mycology in cooperation with the Mycoses Study Group Education and Research Consortium. 2019.

21. Arani R, Shareef SNH, and Khanam HM. Mucormycotic Osteomyelitis Involving the Maxilla: A Rare Case Report and Review of the Literature. Hindawi. Case Reports in Infectious Diseases Volume. 2019.

22. Chakrabarti A, Singh R. "Mucormycosis in India: unique features," Mycoses, 2014; 57: 85-90.

23. Mehta S, Pandey A. Rhino-Orbital Mucormycosis Associated With COVID-19. 2020; 12: 10726.

24. Gangneux JP, Bougnoux ME, Dannaoui E, et al. Invasive fungal diseases during COVID-19: we should be prepared. J Mycol Med. 2020; 30: 100971.

25. The RECOVERY Collaborative Group: Dexamethasone in hospitalized patients with Covid-19 - preliminary report. 2021; 384: 693-704.
26. National Institutes of Health. COVID-19 Treatment Guidelines Panel. Coronavirus disease 2019 (COVID-19) treatment guidelines. 2020.

27. Clinical management protocol for COVID-19. Government of India Ministry of Health and Family Welfare Directorate General of Health Services (EMR Division) Accessed. 2020.

28. Rawson TM, Moore L, Zhu N, et al. Bacterial and fungal coinfection in individuals with coronavirus: a rapid review to support COVID-19 antimicrobial prescribing. Clin Infect Dis. 2020; 71: 2459-2468.

29. Philip Lewis White, Rishi Dhillon, Alan Cordey, et al. A national strategy to diagnose COVID-19 associated invasive fungal disease in the ICU. Clin Infect Dis. 2020.

30. Chowdhary A, Tarai B, Singh A, et al. Multidrug-resistant Candida auris infections in critically ill coronavirus disease patients, India, April-July 2020. Emerg Infect Dis. 2020; 26.

31. Nasir N, Farooqi J, Mahmood SF, et al. COVID-19 associated pulmonary aspergillosis (CAPA) in patients admitted with severe COVID-19 pneumonia: an observational study from Pakistan. Mycoses. 2020; 63: 766-770.

32. Bartoletti M, Pascale R, Cricca M, et al. Epidemiology of invasive pulmonary aspergillosis among COVID-19 intubated patients: a prospective study. Clin Infect Dis. 2020.

33. Hanley B, Kikkeri N, Candice N, et al. Histopathological findings and viral tropism in UK patients with severe fatal COVID-19: a post-mortem study. Lancet Microbe. 2020; 6: 245-253.

34. Werthman-Ehrenreich A: Mucormycosis with orbital compartment syndrome in a patient with COVID-19. Am J Emerg Med. 2020; 42.

35. Roden MM, Zaoutis TE, Buchanan WL, et al. Epidemiology and outcome of zygomycosis: a review of 929 reported cases. Clin Infect Dis 2005; 41: 634-53.

36. Guinea j, Escribano P, Vena A, et al. Increasing incidence of mucormycosis in a large Spanish hospital from 2007 to 2015: Epidemiology and microbiological characterization of the isolates. PLoS One. 2017; 12: 0179136.

37. Marty FM, Zeichner LO, Cornely OA, et al. Isavuconazole treatment for mucormycosis: a single-arm open-label trial and case-control analysis. Lancet Infect Dis 2016; 16: 828-37.

38. Shoham S, Magill SS, Merz WG, etal. Primary treatment of zygomycosis with liposomal amphotericin B: analysis of 28 cases. Med Mycol 2010; 48: 511-517.

39. Legrand M, Muselli MG, Boutin L, et al. Detection of circulating mucorales DNA in critically ill burn patients: preliminary report of a screening strategy for early diagnosis and treatment. Clin Infect Dis. 2016; 63: 1312-1317. 